\title{
A Goblet (Cell) Half Full: What Do We Really Know About Barrett's Esophagus-A Tribute to Emmet Keeffe
}

\author{
Robert S. Bresalier ${ }^{1} \cdot$ Rhonda F. Souza $^{2}$ \\ Published online: 9 June 2018 \\ (c) Springer Science+Business Media, LLC, part of Springer Nature 2018
}

\section{Editors' Introduction}

It is our pleasure to introduce this fourth annual special supplement to Digestive Diseases and Sciences (DDS) dedicated to its former Editor-in-Chief, the late, great Emmet B. Keeffe. Through our many interactions with Emmet, either as Associate Editor (RSB) or as President of the Gastroenterology Research Group (RFS), which named Digestive Diseases and Sciences its official journal (a relationship enthusiastically encouraged and promoted by Emmet), we witnessed first-hand Emmet's tireless efforts to create a journal relevant to both clinicians and scientists. In 2007 Emmet called me (RSB) to ask if I might serve as an Associate Editor for DDS as he had been recently tasked to "reinvent" the journal. His idea was that while other excellent gastroenterology-based journals existed, DDS could fill a niche as a truly translational journal with appeal to a broad audience. I had known Emmet for many years and knew him to be a true Renaissance man, clinician, scientist, and consummate educator and communicator. Needless to say I jumped at the chance to work with him on this exciting project. Under his leadership and that of the current Editor-in-Chief, Jonathan Kaunitz, articles received per year have doubled to over 2000, usage increased greater than 4.5 -fold $(617,734$ in 2017), page count increased 1.5-fold, editorials published increased 15-fold, and impact factor doubled, while time to first decision was reduced from 67 to 23 days. We are honored to Guest Edit this Special Issue devoted to Barrett's esophagus. We recruited authors who are not only experts in the field, but who also have active research endeavors in the areas of pathology, endoscopy, epidemiology, and molecular biology of Barrett's esophagus. The emphasis is not only

Robert S. Bresalier

rbresali@mdanderson.org

1 University of Texas MD Anderson Cancer Center, Houston, TX, USA

2 Baylor University Medical Center, Dallas, TX, USA on the state of the art of what we know, but how ideas are evolving to answer the many gaps in our knowledge [hence a goblet (cell) half full]. We are confident that Emmet would be pleased with the quality, scope, and utility of this Special Issue, and we hope that our readers will be as well.

\section{Clinical and Endoscopic Advances in Barrett's Esophagus}

In Western countries, the rate of increase in the incidence of adenocarcinoma of the esophagus during the past four decades has been among the highest for any cancer. Barrett's esophagus (BE) as the only known precursor to esophageal adenocarcinoma has been a source of great interest and research among epidemiologists, gastroenterologists, oncologists, and surgeons, and fear among the public at large given the common occurrence of gastroesophageal reflux. A dilemma is that while BE is common in North America and Europe (1-2\% of the general adult population and up to $15 \%$ of individuals with frequent gastroesophageal reflux symptoms), the evolution to carcinoma occurs at a rate of between 0.12 and $0.33 \%$ per year $(0.2-0.5 \%$ in other studies) in individuals with $\mathrm{BE}$, and the natural history of evolution to carcinoma is variable. "Barrett's esophagus and esophageal carcinoma: How common are they really?" is the topic of a review by Dr. Aaron Thrift in this issue, while Drs. Kuipers and Spaander discuss the natural history of BE. This then begs the question of in who and how often should we perform screening and surveillance for BE and is it actually worthwhile (Drs. Otaki and Iyer), and is it cost-effective (Drs. Inadomi and Saxena). In order to better individualize diagnosis, surveillance, and treatment, it is important to standardize how we report endoscopic results in patients with BE (Drs. Gorrepati and Sharma), and if we are to perform screening and surveillance are non-invasive or minimally invasive high volume methods feasible (Drs. O’Donovan and Fitzgerald).

The evolution of knowledge and clinical experience regarding $\mathrm{BE}$ and increased sophistication in endoscopic 
techniques has revised our thinking regarding diagnosis and management of BE. This has led to updated clinical guidelines on diagnosis and management of BE (reviewed in an article by Drs. Clermont and Falk). Current guidelines now recommend endoscopic intervention for dysplastic $\mathrm{BE}$ (reviewed by Drs. Visrodia, Zakko and Wang) which while highly successful in eradicating BE may not always have a durable result (the natural history of the post-ablation esophagus is reviewed by Drs. Reed and Shaheen). Endoscopic management of early-stage adenocarcinoma of the esophagus may also be an option for some individuals and is reviewed by Drs. Seewald, Ang, Pouw, Bannwart, and Begman. Successful chemoprevention of BE, especially in high-risk groups, could supplement the benefit of surveillance endoscopy by targeting missed lesions, addressing the development of interval lesions, and theoretically slowing the growth of early cancers. The current status of chemoprevention of $\mathrm{BE}$ and esophageal adenocarcinoma is discussed in a review by Dr. Bresalier which emphasizes the limitations of the current literature and the possibilities for the future.

\section{Histological and Biologic Advances in Barrett's Esophagus}

In recent years, there has been an explosion of studies addressing the histology and cellular origins of Barrett's metaplasia. Based on multiple studies, there are 4 potential sources from which Barrett's metaplasia may arise: (1) epithelial progenitor cells native to the esophagus, (2) epithelial progenitors that line the ducts of the esophageal submucosal glands (both discussed by Drs. Zhang and Wang), (3) progenitors from the squamo-columnar junction or gastric cardia (Drs. Sayin, Baumeister, Wang and Quante), and (4) circulating bone marrow-derived progenitors. There has been considerable recent refinement in our understanding of the concepts surrounding cellular plasticity, transdifferentiation, and dedifferentiation, processes which allow mature, differentiated cells to become stem-like and perhaps give rise to Barrett's metaplasia. What is the basic clonal unit that makes up the Barrett's segment, how do clonal expansions occur within the segment, what are the clonal origins of $\mathrm{BE}$, is the Barrett's segment itself clonal, what is the clonal origin of carcinoma in $\mathrm{BE}$, and what is the clonal architecture of the derivative carcinoma itself are all addressed in a review by Sir Nicholas Wright. Whatever is the cell of origin, intestinal cells are not found normally in the esophagus or stomach, and so the development of Barrett's metaplasia must involve some reprogramming in the expression of key developmental transcription factors to produce a columnar-lined esophagus with intestinal features. The origins of gastric and esophageal metaplasia and whether they are "relatives" is also discussed in an article by Drs. Jin and Mills. There remains an international difference of opinion regarding the type of intestinalized epithelium that qualifies for a diagnosis of Barrett's esophagus (the significance of goblet cells is discussed in a review by Dr. Robert Odze, and the significance of cardiac metaplasia is discussed in a review by Dr. Stuart Spechler). European gastroenterologists accept an esophagus lined by columnar epithelium with intestinal features but without goblet cells (i.e., cardiac mucosa) as BE, whereas American gastroenterologists require the presence of goblet cells for a Barrett's diagnosis. We have included reviews addressing all of these conceptual issues and controversies. Among the limitations of the requirement for the histological identification of goblet cells to diagnose BE is biopsy sampling error. Technologic advances in endoscopic imaging [i.e., confocal laser endomicroscopy (CLE)] and improved sampling of Barrett's mucosa (i.e., wide area tissue sampling) might help overcome this limitation. Chapters on screening and surveillance will address technologic advances such as these.

\section{Molecular Advances in Barrett's Esophagus}

Clinicians are well aware of problems in using the histopathological grading of dysplasia to make clinical decisions regarding cancer risk. Amazing advances in "omics" techniques such as whole-genome sequencing, whole-exome sequencing, and genome-wide epigenetic array analyses have revolutionized our understanding of the pathogenesis of $\mathrm{BE}$ and its neoplastic progression, as well as our ability to identify novel biomarkers of neoplastic progression (reviewed by Drs. Grady and $\mathrm{Yu}$ ). Genomic and epigenomic analyses combined with environmental risk factors could be used to develop risk prediction scores with the potential to improve the precision of risk stratification for individual patients. In the past few years, we have seen that biomarker identification need no longer be confined to endoscopic biopsy tissues alone. Biomarkers can now be identified from whole blood, from esophageal cells obtained by tethered capsules, and from volatile organic compounds exhaled in the breath (see article by Drs. Konda and Souza). Moreover, innovations in advanced molecular imaging are complimenting these in vitro analysis by providing immediately available information in vivo that can be molecular (i.e., CLE) or dynamic (optical coherence tomography) in nature. Included chapters will summarize these concepts and provide an update on the current status of laboratory-based and endoscopy-based biomarker advancements. 


\section{Final Comments}

We sincerely hope that you will find this Special Issue comprehensive, informative, and thought-provoking. We cannot offer enough thanks to the authors who have contributed to this issue. Their generosity in time and devotion to their topics would certainly make Emmet proud. We would also like to thank Meghan Keeffe, the Managing Editor of DDS, for helping us with the innumerable organizational details required for a project of this magnitude.

Bob and Rhonda 